\title{
Development of Omnidirectional Collision-Detecting Bumper Using Noncontact Sensors
}

Noriyuki Shimpuku* and Hiroyuki Miyamoto

Graduate School of Life Science and Systems Engineering, Kyushu Institute of Technology, Japan

\begin{abstract}
The omnidirectional mobile mechanism has high movement performance. We developed a prototype omnidirectional collision-detecting bumper using potentiometers for such a mobile mechanism. However, the previous prototype had low durability because it used contact-type sensors and a link mechanism to convert from the displacement of the bumper to the rotation of the potentiometers. In this paper, we propose a new prototype omnidirectional collision-detecting bumper using noncontact-type sensors. In an experiment, the new prototype showed higher accuracy than the previous one.
\end{abstract}

Keywords: Collision detection; Noncontact sensor; Omnidirectional collision-detecting bumper

\section{Introduction}

Omnidirectional mobile vehicles have been studied for a long time because of their high moving performance [1-3]. Omni wheels $[4]$ and Mecanum wheels [5,6] are typically used because they are easy to implement, i.e., they can realize omnidirectional motion merely by replacing the wheels of existing vehicles. However, it is difficult for such wheels to negotiate steps, gaps, and on-slopes because their traveling ability depends on passive rollers. We have developed a ball wheel drive mechanism [7] that uses active-rolling balls as drive wheels, which showed higher climbing ability for steps and gaps and better control on slopes than the conventional omnidirectional wheel [8].

In the case of applying the omnidirectional mobile vehicle as a robot or an electric wheelchair, it is considered that it should have a system that detects and avoids obstacles in any direction to ensure the safety of the mobile vehicle. The conventional methods, for example, the omnidirectional vision system $[9,10]$ and the method using a laser range finder (LRF) [11], are well known and provide accurate solutions but they require expensive sensors and time-consuming calculations. Other conventional methods using IR sensors and ultrasonic sensors $[12,13]$ are less-expensive but they require many sensors and are have low robustness to distance noise.

In our previous study [14], an omnidirectional collision-detecting bumper (Omni Bumper) using three potentiometers was developed as a prototype low-cost collision-detecting device. Figure la shows an overview of the Omni Bumper with the potentiometers. The performance of collision detection by the bumper was shown in three degrees of freedom through a movement experiment.

However, the bumper had a problem of low durability because it used a link mechanism to convert from the displacement of the bumper to the rotation of the potentiometers. Therefore, we developed a new Omni Bumper using non-contact sensor instead of the link mechanism, as shown in Figure $1 \mathrm{~b}$, to solve this problem. The new Omni Bumper uses PSD (Position Sensitive Detector) [15] sensors to measure the displacement of the bumper. In this paper, we present the details of the new bumper and experimental results.

\section{Omni bumper using non-contact sensors}

Figure 2a shows the positional relation between the $i$ th $(i=1, \ldots, 3)$ PSD sensor and the bumper when the bumper is in the initial position.
The center of the bumper is the same as the center of the body $(0,0)$. $\gamma_{i}$ represents the angle from the center of the bumper to the center position of the side frame of the bumper $p_{0 i}$. The position $p_{0 i}$ is $\left(L \cos \gamma_{i}\right.$; $L \sin \gamma_{i}$ ) when the bumper is in the initial position. The ith PSD sensor is placed at the position $\left(x_{0 i}, y_{0 i}\right)$ with angle $\gamma_{i}$ so that it faces the side frame of the bumper. The position $\left(x_{0 i}, y_{0 i}\right)$ can be calculated as

$$
\begin{aligned}
& x_{0 i}=(L-h) \cos \gamma_{i}-e \sin \gamma i \\
& y_{0 i}=(L-h) \sin \gamma_{i}+e \cos \gamma i
\end{aligned}
$$

The broken line from the position $\left(x_{0 i}, y_{0 i}\right)$ to the side of the bumper represents the measurement line along which the infrared ray of the PSD sensor passes. An arbitrary point on the broken line satisfies

$$
\left(y_{i}-y_{0 i}\right)=\left(x_{i}-x_{0 i}\right) \tan y_{i}
$$

because the line passes through the position $\left(x_{0 i}, y_{0 i}\right)$ and its inclination is $y_{i}$.di denotes the distance from the position $\left(x_{0 i}, y_{0 i}\right)$ to the position $\left(x_{i}, y_{i}\right)$ on this line. $x_{i}$ and $y_{i}$ are calculated as functions of $d_{i}$ as

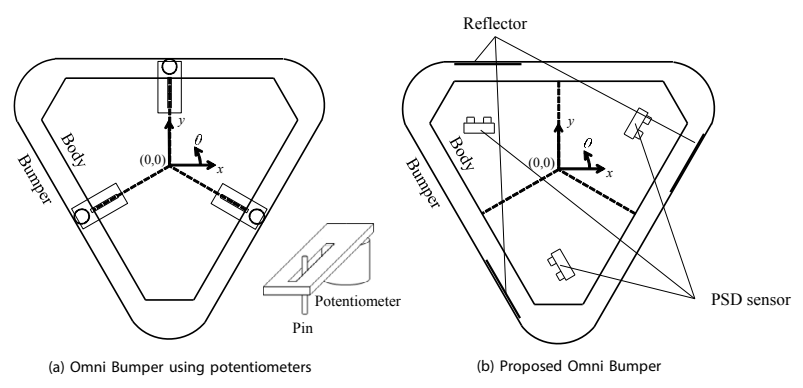

Figure 1: Omni Bumper.

*Corresponding author: Noriyuki Shimpuku, Graduate School of Life Science and Systems Engineering, Kyushu Institute of Technology, Japan, Tel: 81-093-69561; E-mail: shinpuku-noriyuki@edu.brain.kyutech.ac.jp

Received August 16, 2014; Accepted September 19, 2014; Published September 21,2014

Citation: Shimpuku N, Miyamoto H (2014) Development of Omnidirectional Collision-Detecting Bumper Using Noncontact Sensors. J Comput Sci Syst Biol 7: 221-225. doi: $10.4172 / \mathrm{jcsb} .1000160$

Copyright: @ 2014 Shimpuku N, et al. This is an open-access article distributed under the terms of the Creative Commons Attribution License, which permits unrestricted use, distribution, and reproduction in any medium, provided the original author and source are credited. 


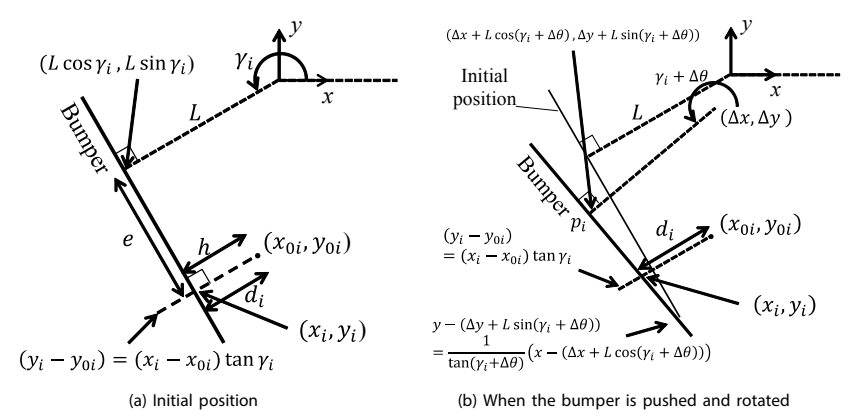

Figure 2: Positional relationships in the system.

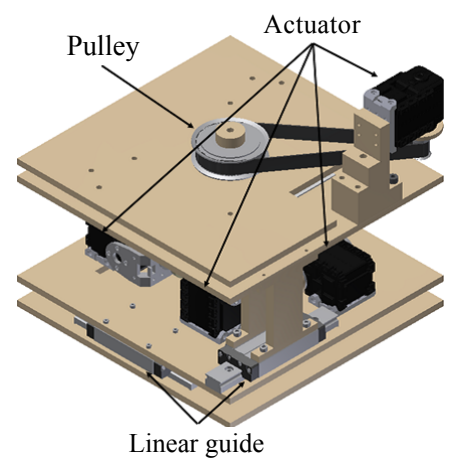

Figure 3: Positioning device.

$$
\begin{aligned}
& x_{i}=x_{0 i}+d_{i} \cos y_{i} \\
& y_{i}=y_{0 i}+d_{i} \sin y_{i} .
\end{aligned}
$$

Figure $2 \mathrm{~b}$ shows the positional relation when the bumper is pushed and rotated by an obstacle and the position of the bumper is changed from the initial position. In this case, the position $p i$, which is the current center position of the side frame of the bumper, becomes $\left(\Delta x+L \cos \left(\gamma_{i}+\Delta \theta\right), \Delta y+L \sin \left(y_{i}+\Delta \theta\right)\right)$. An arbitrary point on the side frame of the bumper $\left(x_{i} ; y_{i}\right)$ satisfies

$$
y_{i}-\left(\Delta y+L \sin \left(\gamma_{i}+\Delta \theta\right)\right)=-\frac{1}{\tan \left(\gamma_{i}+\Delta \theta\right)}\left(x_{i}-\left(\Delta x+L \cos \left(\gamma_{i}+\Delta \theta\right)\right)\right)
$$

The $x_{i}$ and $y_{i}$ obtained from Eq. (3) are used in Eq. (4). We apply an approximation to Eq. (4) since it is otherwise impossible to solve. We use the addition theorem to expand the trigonometric functions in Eq. (4) and assume $\Delta x \ll 1, \Delta y \ll 1, \Delta \theta \ll 1$ and replace $\tan \Delta \theta \fallingdotseq \Delta \theta, \sin \Delta \theta \fallingdotseq \Delta \theta, \cos \Delta \theta \fallingdotseq 1$. Moreover, assuming that $\Delta x \Delta \theta$ and $\Delta y \Delta \theta$ can be ignored, we obtain

$$
\cos \gamma_{i} \Delta x-\sin \gamma_{i} \Delta y-\left(\sin \gamma_{i} x_{i}-\cos \gamma_{i} y_{i}\right) \Delta \theta=\cos \gamma_{i} x_{i}+\sin \gamma_{i} y_{i}-L
$$

According to Figure $2 \mathrm{~b}, \gamma_{1}=\pi / 2, \gamma_{2}=\pi / 2+2 \pi / 3$ and $\gamma_{3}=\pi / 2+4 \pi / 3$. Thus, from Eq. (5), we have the following simultaneous linear equations containing three unknowns, $\Delta x, \Delta y$ and $\Delta \theta$.

$$
\begin{aligned}
& \Delta y-x_{1} \Delta \theta=L-y_{1} \\
& \sqrt{3} \Delta x-\Delta y+\left(x_{2}-\sqrt{3} y_{2}\right) \Delta \theta \Delta y+\left(x_{2}-\sqrt{3} y_{2}\right) \\
& -\sqrt{3} \Delta x-\Delta y+\left(x_{3}-\sqrt{3} y_{3}\right) \Delta y+\left(x_{3}-\sqrt{3} y_{3}\right)
\end{aligned}
$$

These equations can be expressed as

$A b=c$,

Where,

$$
\begin{aligned}
& A=\left(\begin{array}{ccc}
0 & 1 & -x_{1} \\
\sqrt{3} & -1 & x_{2}-\sqrt{3} y_{2} \\
\sqrt{3} & -1 & x_{3}+\sqrt{3} y_{3}
\end{array}\right), \\
& b=(\Delta x \Delta y \Delta \theta)^{\mathrm{T}} \\
& c=\left(\begin{array}{l}
-L-y_{1} \\
2 L+\sqrt{3} x_{2}+y_{2} \\
2 L-\sqrt{3} x_{3}+y_{3}
\end{array}\right) .
\end{aligned}
$$

If $A^{-1}$ exists $\Delta x, \Delta y$ and $\Delta \theta$ can be calculated as

$$
b=A^{-1} c
$$

$$
\mathrm{A}^{-1}=\frac{1}{2 u+v+w}\left(\begin{array}{ccc}
\frac{w-v}{\sqrt{3}} & \frac{u+w}{\sqrt{3}} & \frac{-u-v}{\sqrt{3}} \\
v+w & -u & -u \\
2 & 1 & 1
\end{array}\right)
$$

where

$$
\begin{aligned}
& u=-x_{1}, \\
& v=x_{2}-\sqrt{3} y_{2}, \\
& w=x_{3}+\sqrt{3} y_{3} .
\end{aligned}
$$

According to the positional relationship between the sensors shown in Figure $1 \mathrm{~b}$, $\operatorname{det} A=2 u+v+w \neq 0$.

\section{Experiments}

\section{Positioning device}

It is important to evaluate the accuracy of the new Omni Bumper. In the experiment in our previous study [14], an external force was applied manually to the bumper. However, it is considered that conducting an experiment manually takes a long time and the result may contain human error.

Therefore, we also developed an automatic positioning device to decrease human error and perform the experiment effectively. Figure 3 shows an overview of the positioning device and Table 1 shows the specifications of the positioning device.

\section{Experiment to determine accuracy}

Using the positioning device, the displacement detecting accuracy of the new Omni Bumper was evaluated. Figure 4 shows the Omni Bumper with the positioning device. In this experiment, the springs under the bumper were removed. The positioning device automatically moved the bumper by $\pm 15 \mathrm{~mm}$ in $0.1 \mathrm{~mm}$ intervals in the $x$ and $y$ directions and rotated the bumper by $\pm 7 \mathrm{deg}$ in $0.1 \mathrm{deg}$ intervals in the $\theta$ direction.

Figures 5-7 show the results of the experiment. The horizontal axis represents the target position, the vertical axis represents the measured 


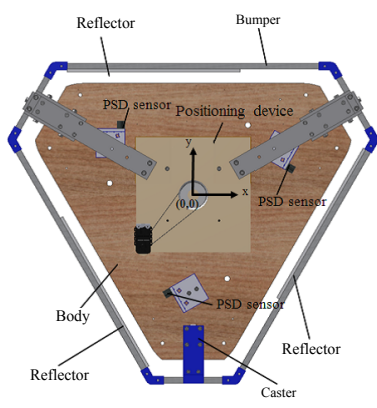

(a) Top view

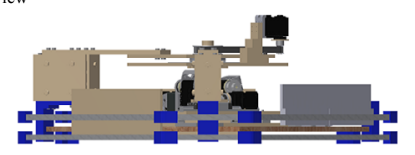

(c) Side view

Figure 4: New Omni Bumper with positioning device.

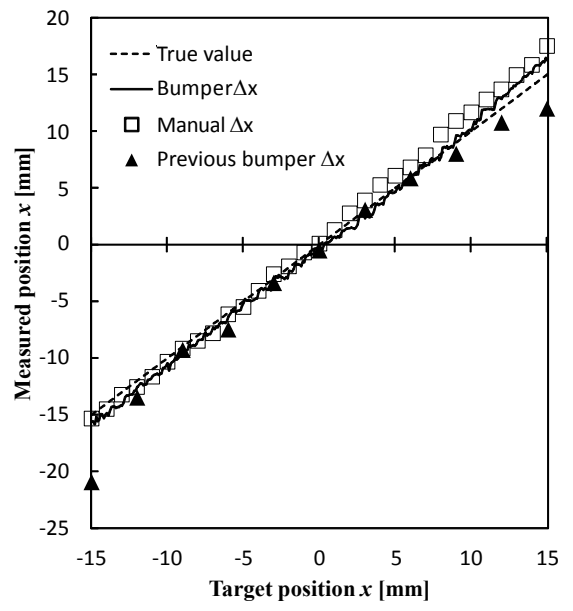

Figure 5: Results in $x$ direction.

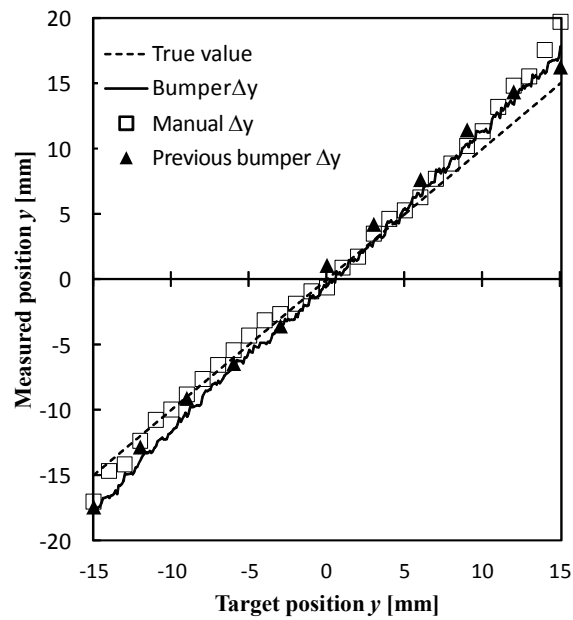

Figure 6: Results in $y$ direction.

position, the solid line represents the measured values obtained by the bumper, and the broken line represents the amount of movement of

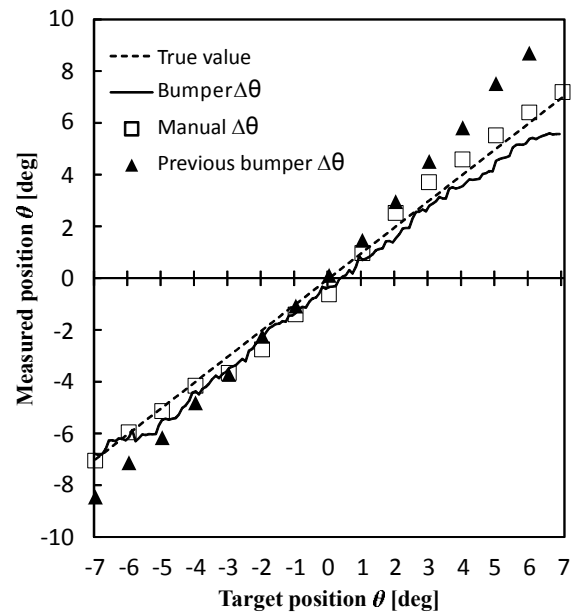

Figure 7: Results in $\theta$ direction.

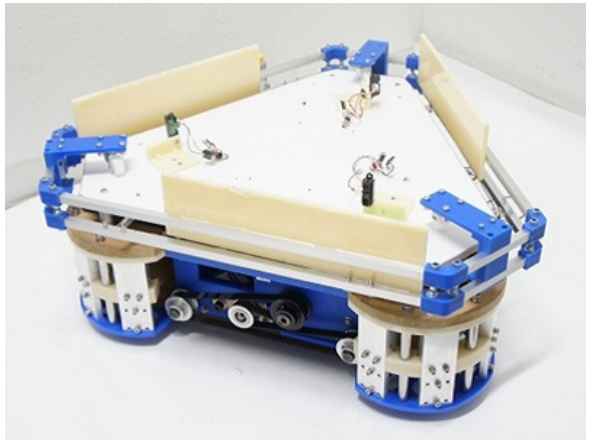

Figure 8: Omni directional mobile vehicle with new Omni Bumper.

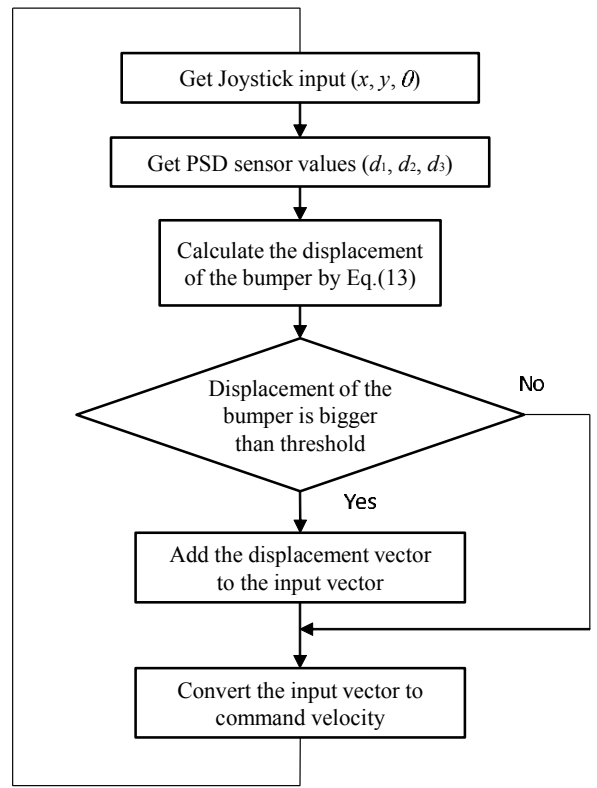

Figure 9: Flowchart of collision avoidance.

the positioning device in each figure. To enable comparison with the previous prototype Omni Bumper, the experimental results from [14] 


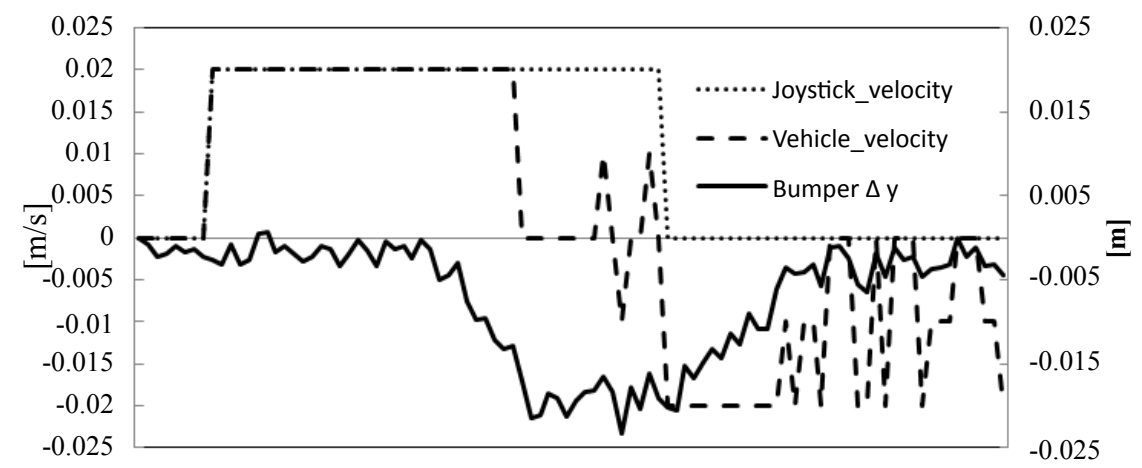

Figure 10: Results of collision-avoidance experiment.

\begin{tabular}{|l|l|}
\hline Width & Width $200[\mathrm{~mm}]$ \\
\hline Depth & Depth $200[\mathrm{~mm}]$ \\
\hline Height & Height $168[\mathrm{~mm}]$ \\
\hline Weight & Weight $2.5[\mathrm{~kg}]$ \\
\hline Actuator & Robotis Dynamixel AX-12×5 \\
\hline
\end{tabular}

Table 1: Specifications of positioning device.

\begin{tabular}{|l|l|}
\hline Width & $578.6[\mathrm{~mm}]$ \\
\hline Length & $529.1[\mathrm{~mm}]$ \\
\hline Weight & $26[\mathrm{~kg}]$ \\
\hline Ball diameter & $98[\mathrm{~mm}]$ \\
\hline Ball weight & $0.56[\mathrm{~kg}]$ \\
\hline Ball material & Urethane shore 90 \\
\hline Rotor diameter & $40[\mathrm{~mm}]$ \\
\hline Rotor material & Stainless steel (SUS304) \\
\hline Actuator & Oriental DC 100 [W] $\times 3$ \\
\hline Gear ratio & $15: 1$ \\
\hline
\end{tabular}

Table 2: Specifications of omnidirectional mobile vehicle.

(triangles) are plotted.

The measurements of the new Omni Bumper obtained manually (squares) are also plotted to confirm the effect of the positioning device. The maximum errors of the new Omni Bumper when using the positioning device are $x: 1.58 \mathrm{~mm}, y: 2.97 \mathrm{~mm}$ and $\theta: 1.33 \mathrm{deg}$, and the maximum errors of the new Omni Bumper without the positioning device are $x: 2.48 \mathrm{~mm}, y: 4.68 \mathrm{~mm}$ and $\theta: 0.76 \mathrm{deg}$. The maximum errors of the previous Omni Bumper are $x: 5.95 \mathrm{~mm}, y: 2.45 \mathrm{~mm}$ and $\theta: 3.43$ deg. The new Omni Bumper has equal or higher accuracy than the previous bumper.

\section{Experiment on collision avoidance}

Figure 8 shows an overview of the new Omni Bumper attached to the omnidirectional mobile vehicle with a ball wheel drive mechanism and Table 2 shows the specifications of the omnidirectional mobile vehicle.

In this experiment, the omnidirectional mobile vehicle was moved forward by manipulating a joystick until the bumper collided with an obstacle. Figure 9 shows a flowchart of the collision avoidance algorithm and Figure 10 shows the result of the experiment. On the basis of the displacement of the Omni Bumper, the omnidirectional mobile vehicle stopped and then moved backward when there was no input via the joystick.

\section{Discussion}

It is considered that the new Omni Bumper produced better results than the previous bumper because the error due to the distortion of parts of the bumper was reduced. Although the new Omni Bumper, unlike the conventional methods, cannot detect multiple obstacles, this is not considered a problem because the result of collisions is manifested as a displacement of the bumper. Since it is intended to be used in indoor environments, the movable range of the bumper is considered sufficient. With the use of the positioning device, the total number of samples was increased tenfold and the total time taken to measure all axes changed from about $90 \mathrm{~min}$ to about $13 \mathrm{~min}$.

\section{Conclusion}

In this study, we developed a new Omni Bumper with noncontact sensors as an Omni directional collision-detecting device. We also developed a positioning device to evaluate the accuracy of the new Omni Bumper. The experimental results show:

- The new Omni-Bumper was accurate equal or more than the previous one.

- The omni-directional mobile vehicle with the new Omni Bumper could detect a collision with an obstacle and it stopped and went back according to the displacement of the bumper.

Our future work is to improve the accuracy and expand the measuring range of the Omni Bumper.

\section{References}

1. Carlisle B (1983) An omni-directional mobile robot: Development in Robotics. IFS Publications, Kempston.

2. Pin FG, Killough SM (1994) A new family of omnidirectional and holonomic wheeled platforms for mobile robots. IEEE Transactions on Robotics and Automation 10: 480-489.

3. Tadakuma K, Tadakuma R, Berengeres J (2007) Development of holonomic omnidirectional vehicle with "Omni-Ball": spherical wheels. Proceedings of 2007 IEEE/RSJ International Conference on Intelligent Robots and Systems 33-39.

4. Asama H, Sato M, Bogoni L, Kaetsu H, Matsumoto A, et al. (1995) Development of an omni-directional mobile robot with 3 DOF decoupling drive mechanism. Proceedings of IEEE International Conference on Robotics and Automation 2: 1925-1930.

5. Ilon BE (1975) Wheels for a course stable self-propelling vehicle movable in any desired direction on the ground or some other base. United States Patent $3,876,255$. 
Citation: Shimpuku N, Miyamoto H (2014) Development of Omnidirectional Collision-Detecting Bumper Using Noncontact Sensors. J Comput Sci Syst Biol 7: 221-225. doi:10.4172/jcsb.1000160

6. Diegel O, Badve A, Bright G, Potgieter J, Tlale S (2002) Improved mecanum wheel design for omni-directional robots. Proceedings of 2002 Australasian Conference on Robotics and Automation.

7. Ishida S, Miyamoto H (2012) Holonomic omni-directional vehicle with ball wheel drives mechanism. Transactions of the Japan Society of Mechanical Engineering Series C 78: 2162-2170.

8. Ishida S, Shimpuku N, Ishii K, Miyamoto H (2014) Holonomic omnidirectional vehicle with ball wheel drive mechanism and application to RoboCup soccer middle size league. Journal of Japan Society for Fuzzy Theory and Intelligent Informatics 26: 669-677.

9. Yagi Y, Kawato S, Tsuji S (1994) Real time omnidirectional image sensor (COPIS) for vision-guided navigation. IEEE Transactions of Robotics and Automation 10: 11-22.
10. Satoh Y, Sakaue K (2007) An omnidirectional stereo vision-based smart wheelchair. Journal on Image and Video Processing 2007: 087646.

11. Prassler E, Scholz J, Fiorini $P$ (2001) A robotic wheelchair for crowded public environments. IEEE Robotics Automation Magazine 8: 38-45.

12. Miller DP, Slack MG (1995) Design and testing of a low-cost robotic wheelchair prototype. Autonomous Robots 2: 77-88.

13. Lankenau A, Rofer T (2001) A versatile and safe mobility assistant. IEEE Robotics Automation Magazine 8: 29-37.

14. Ishida S, Miyamoto H (2013) Collision- detecting device for omnidirectional electric wheelchair. Robotics 2013: 1-8.

15. Sharp GP2Y0A21YK. 